\title{
MULTIPLE SOLUTIONS FOR THE $p$-LAPLACE OPERATOR WITH CRITICAL GROWTH
}

\author{
PABLO L. DE NÁPOLI, JULIÁN FERNÁNDEZ BONDER \& ANALÍA SILVA
}

\begin{abstract}
In this paper we show the existence of at least three nontrivial solutions to the following quasilinear elliptic equation $-\Delta_{p} u=|u|^{p^{*}-2} u+$ $\lambda f(x, u)$ in a smooth bounded domain $\Omega$ of $\mathbb{R}^{N}$ with homogeneous Dirichlet boundary conditions on $\partial \Omega$, where $p^{*}=N p /(N-p)$ is the critical Sobolev exponent and $\Delta_{p} u=\operatorname{div}\left(|\nabla u|^{p-2} \nabla u\right)$ is the $p$-laplacian. The proof is based on variational arguments and the classical concentration compactness method.
\end{abstract}

\section{INTRODUCTION.}

Let us consider the following nonlinear elliptic problem:

$$
\begin{cases}-\Delta_{p} u=|u|^{p^{*}-2} u+\lambda f(x, u) & \text { in } \Omega \\ u=0 & \text { on } \partial \Omega,\end{cases}
$$

where $\Omega$ is a bounded smooth domain in $\mathbb{R}^{N}, \Delta_{p} u=\operatorname{div}\left(|\nabla u|^{p-2} \nabla u\right)$ is the $p$-laplacian, $1<p<N, p^{*}=N p /(N-p)$ is the critical exponent in the Sobolev embedding and $\lambda$ is a positive parameter.

Problems like $(\mathrm{P})$ appears naturally in several branches of pure and applied mathematics, such as the theory of quasiregular and quasiconformal mappings in Riemannian manifolds (see [10, 22 ]), non-Newtonian fluids, reaction diffusion problems, flow through porous media, nonlinear elasticity, glaciology, etc. (see [1, 2, 3, 8]).

The purpose of this paper, is to prove the existence of at least three nontrivial solutions for $(\mathbb{P})$ under adequate assumptions on the source term $f$ and the parameter $\lambda$. This result extends an old paper by Struwe [21]. A related result for the nonlinear boundary condition case can be found in 11 .

Here, no oddness condition is imposed in $f$ and a positive, a negative and a signchanging solution are found (For odd nonlinearities it is well known that infinitely many solutions can be obtained in many situations, by using the tools of critical point theory, as the $\mathbb{Z}_{2}$-symmetric version of the mountain pass theorem. See for instance [19] or 9]).

The proof of our result relies on the variational principle of Ekeland (see [20]) complemented with the, by now, well known concentration compactness method of P.L. Lions (see [18).

Key words and phrases. p-laplace equations, critical growth, variational methods.

2000 Mathematics Subject Classification. 35J60, 35J20.

Supported by Universidad de Buenos Aires under grant X078 and X837, by ANPCyT PICT No. 2006-290 and CONICET (Argentina) PIP 5477 and PIP 5478/1438. J. Fernández Bonder and Pablo De Nápoli are members of CONICET. Analía Silva is a fellow of CONICET.. 
One of the advantages in using Ekeland's variational principle is that it allows to split the geometry of the problem from the compactness aspect of it. This approach simplifies the one applied by M. Struwe in [21] for the subcritical case.

The use of the concentration compactness method to deal with the $p$-Laplacian has been used by several authors before. One of the first results in this direction was obtained by J. García Azorero and I. Peral in [16]. Here, we borrow some ideas from that work.

For related results with subcritical growth, see the already mentioned [21] and more recently $[4,23$.

Also, for related results with critical growth, but with subcritical power perturbation, see the seminal work of M. Guedda and L. Veron [17] and, more recently, the paper by S. Cingolani and G. Vannella [5].

Throughout this work, by (weak) solutions of $(\mathrm{P})$ we understand critical points of the associated energy functional acting on the Sobolev space $W_{0}^{1, p}(\Omega)$ :

$$
\Phi(v)=\frac{1}{p} \int_{\Omega}|\nabla v|^{p} d x-\frac{1}{p^{*}} \int_{\Omega}|u|^{p^{*}} d x-\lambda \int_{\Omega} F(x, v) d x,
$$

where $F(x, u)=\int_{0}^{u} f(x, z) d z$.

We will denote

$$
\mathcal{F}_{\lambda}(v)=\int_{\Omega} \frac{1}{p^{*}}|v|^{p^{*}}+\lambda F(x, v) d x,
$$

so the functional $\Phi$ can be rewritten as

$$
\Phi(v)=\frac{1}{p}\|v\|_{W^{1, p}(\Omega)}^{p}-\mathcal{F}_{\lambda}(v) .
$$

\section{Assumptions and statement of the Results.}

The precise assumptions on the source terms $f$ are as follows:

(F1) $f: \Omega \times \mathbb{R} \rightarrow \mathbb{R}$, is a measurable function with respect to the first argument and continuously differentiable with respect to the second argument for almost every $x \in \Omega$. Moreover, $f(x, 0)=0$ for every $x \in \Omega$.

(F2) There exist constants $c_{1} \in\left(0,1 /\left(p^{*}-1\right)\right), k_{2} \in\left(p, p^{*}\right), 0<c_{3}<c_{4}$, such that for any $u \in L^{q}(\Omega)$ and $p<q<p^{*}$.

$$
\begin{aligned}
c_{3}\|u\|_{L^{q}(\Omega)}^{q} & \leq k_{2} \int_{\Omega} F(x, u) d x \leq \int_{\Omega} f(x, u) u d x \\
& \leq c_{1} \int_{\Omega} f_{u}(x, u) u^{2} d x \leq c_{4}\|u\|_{L^{q}(\Omega)}^{q}
\end{aligned}
$$

Remark 1. Observe that this set of hypotheses on the nonlinear term $f$ are weaker than the ones considered by [20].

Remark 2. We exhibit now two families of examples of nonlinearities that fulfill all of our hypotheses.

- $f(x, u)=|u|^{q-2} u+\left|u_{+}\right|^{r-2} u_{+}$, if $r \leq q$.

Hypotheses (F1)-(F2) are clearly satisfied. 
- $f(x, u)=\left\{\begin{array}{ll}|u|^{q-2} u+|u|^{r-2} u & u \geq 0 \\ |u|^{q-2} u+|u|^{r-2} u & u<0\end{array}\right.$, if $r \leq q$

Hypotheses (F1)-(F2) are, again, clearly satisfied.

So the main result of the paper reads:

Theorem 1. Under assumptions (F1)-(F2), there exist $\lambda^{*}>0$ depending only on $n, p, q$ and the constant $c_{3}$ in $(\mathrm{F} 2)$, such that for every $\lambda>\lambda^{*}$, there exists three different, nontrivial, (weak) solutions of problem (프. Moreover these solutions are, one positive, one negative and the other one has non-constant sign.

\section{Proof of Theorem 1.}

The proof uses the same approach as in 21]. That is, we will construct three disjoint sets $K_{i} \neq \emptyset$ not containing 0 such that $\Phi$ has a critical point in $K_{i}$. These sets will be subsets of $C^{1}$-manifolds $M_{i} \subset W^{1, p}(\Omega)$ that will be constructed by imposing a sign restriction and a normalizing condition.

In fact, let

$M_{1}=\left\{u \in W_{0}^{1, p}(\Omega): \int_{\Omega} u_{+}>0\right.$ and $\left.\int_{\Omega}\left|\nabla u_{+}\right|^{p}-\left|u_{+}\right|^{p^{*}} d x=\int_{\Omega} \lambda f(x, u) u_{+} d x\right\}$,

$M_{2}=\left\{u \in W_{0}^{1, p}(\Omega): \int_{\Omega} u_{-}>0\right.$ and $\left.\int_{\Omega}\left|\nabla u_{-}\right|^{p}-\left|u_{-}\right|^{p^{*}} d x=\int_{\Omega} \lambda f(x, u) u_{-} d x\right\}$,

$M_{3}=M_{1} \cap M_{2}$.

where $u_{+}=\max \{u, 0\}, u_{-}=\max \{-u, 0\}$ are the positive and negative parts of $u$, and $\langle\cdot, \cdot\rangle$ is the duality pairing of $W^{1, p}(\Omega)$.

Finally we define

$$
\begin{aligned}
& K_{1}=\left\{u \in M_{1} \mid u \geq 0\right\}, \\
& K_{2}=\left\{u \in M_{2} \mid u \leq 0\right\}, \\
& K_{3}=M_{3} .
\end{aligned}
$$

First, we need a Lemma to show that these sets are nonempty and, moreover, give some properties that will be useful in the proof of the result.

Lemma 1. For every $w_{0} \in W_{0}^{1, p}(\Omega), w_{0}>0\left(w_{0}<0\right)$, there exists $t_{\lambda}>0$ such that $t_{\lambda} w_{0} \in M_{1}\left(\in M_{2}\right)$. Moreover, $\lim _{\lambda \rightarrow \infty} t_{\lambda}=0$.

As a consequence, given $w_{0}, w_{1} \in W_{0}^{1, p}(\Omega), w_{0}>0, w_{1}<0$, with disjoint supports, there exists $\bar{t}_{\lambda}, \underline{t}_{\lambda}>0$ such that $\bar{t}_{\lambda} w_{0}+\underline{t}_{\lambda} w_{1} \in M_{3}$. Moreover $\bar{t}_{\lambda}, \underline{t}_{\lambda} \rightarrow 0$ as $\lambda \rightarrow \infty$.

Proof. We prove the lemma for $M_{1}$, the other cases being similar.

For $w \in W_{0}^{1, p}(\Omega), w \geq 0$, we consider the functional

$$
\varphi_{1}(w)=\int_{\Omega}|\nabla w|^{p}-|w|^{p^{*}}-\lambda f(x, w) w d x .
$$

Given $w_{0}>0$, in order to prove the lemma, we must show that $\varphi_{1}\left(t_{\lambda} w_{0}\right)=0$ for some $t_{\lambda}>0$. Using hypothesis (H3), we have that:

$$
\varphi_{1}\left(t w_{0}\right) \geq A t^{p}-B t^{p^{*}}-\lambda c_{4} C t^{q}
$$


and

$$
\varphi_{1}\left(t w_{0}\right) \leq A t^{p}-B t^{p^{*}}-\lambda c_{3} C t^{q},
$$

where the coefficients $A, B$ and $C$ are given by:

$$
A=\int_{\Omega}\left|\nabla w_{0}\right|^{p} d x, \quad B=\int_{\Omega}\left|w_{0}\right|^{p^{*}} d x, \quad C=\int_{\Omega}\left|w_{0}\right|^{q} d x .
$$

Since $p<q<p^{*}$ it follows that $\varphi_{1}\left(t w_{0}\right)$ is positive for $t$ small enough, and negative for $t$ big enough. Hence, by Bolzano's theorem, there exists some $t=t_{\lambda}$ such that $\varphi_{1}(t \lambda u)=0$. (This $t_{\lambda}$ needs not to be unique, but this does not matter for our purposes).

In order to give an upper bound for $t_{\lambda}$, it is enough to find some $t_{1}$, such that $\varphi_{1}\left(t_{1} w_{0}\right)<0$. We observe that:

$$
\varphi_{1}\left(t w_{0}\right) \leq A t^{p}-\lambda c_{3} C t^{q} .
$$

so it is enough to choose $t_{1}$ such that $A t_{1}^{p}-\lambda c_{3} C t_{1}^{q}=0$, i.e.,

$$
t_{1}=\left(\frac{A}{c_{3} \lambda C}\right)^{1 /(q-p)} .
$$

Hence, again by Bolzano's theorem, we can choose $t_{\lambda} \in\left[0, t_{1}\right]$, which implies that $t_{\lambda} \rightarrow 0$ as $\lambda \rightarrow+\infty$.

For the proof of the Theorem, we need also the following Lemmas.

Lemma 2. There exist $c_{j}>0$ such that, for every $u \in K_{i}, i=1,2,3$,

$$
\int_{\Omega}|\nabla u|^{p} d x \leq C_{1}\left(\lambda \int_{\Omega} f(x, u) u d x+\int_{\Omega}|u|^{p^{*}} d x\right) \leq C_{2} \Phi(u) \leq C_{3}\left(\int_{\Omega}|\nabla u|^{p} d x\right) .
$$

Proof. As $u \in K_{i}$, we have that

$$
\int_{\Omega}|\nabla u|^{p} d x=\int_{\Omega} \lambda f(x, u) u+|u|^{p^{*}} d x .
$$

This proves the first inequality.

Now, by (F3)

$$
\int_{\Omega} F(x, u) d x \leq \frac{1}{c_{2}} \int_{\Omega} f(x, u) u d x .
$$

Furthermore,

$$
\begin{aligned}
\left|\lambda \int_{\Omega} F(x, u) d x\right|=\lambda \int_{\Omega} F(x, u) d x & \leq \frac{1}{c_{2}} \int_{\Omega} \lambda f(x, u) u d x \\
& =\frac{1}{c_{2}}\left(\int_{\Omega}|\nabla u|^{p}-|u|^{p^{*}} d x\right),
\end{aligned}
$$

so,

$$
-\lambda \int_{\Omega} F(x, u) d x \leq \frac{1}{c_{2}}\left(\int_{\Omega}|\nabla u|^{p}-|u|^{p^{*}} d x\right) .
$$


By (3), we have:

$$
\begin{aligned}
\Phi(u) & =\int_{\Omega} \frac{1}{p}|\nabla u|^{p}-\frac{1}{p^{*}}|u|^{p^{*}}-\lambda F(x, u) d x \\
& \leq \int_{\Omega} \frac{1}{p}|\nabla u|^{p}-\frac{1}{p^{*}}|u|^{p^{*}} d x+\frac{1}{c_{2}}\left(\int_{\Omega}|\nabla u|^{p}-|u|^{p^{*}} d x\right) \\
& \leq\left(\frac{1}{c_{2}}+\frac{1}{p}\right) \int_{\Omega}|\nabla u|^{p} d x .
\end{aligned}
$$

This proves the third inequality.

To prove the middle inequality we proceed as follows:

$$
\begin{aligned}
\Phi(u) & =\int_{\Omega} \frac{1}{p}|\nabla u|^{p}-\frac{1}{p^{*}}|u|^{p^{*}}-\lambda F(x, u) d x \geq \int_{\Omega} \frac{1}{p}\left(|\nabla u|^{p}-|u|^{p^{*}}\right)-\lambda F(x, u) d x \\
& \geq \frac{1}{p} \int_{\Omega} \lambda f(x, u) u d x-\lambda \int_{\Omega} F(x, u) d x \geq\left(\frac{1}{p}-\frac{1}{c_{2}}\right) \lambda \int_{\Omega} f(x, u) d x .
\end{aligned}
$$

This finishes the proof.

Lemma 3. There exists $c>0$ such that

$$
\begin{aligned}
\left\|\nabla u_{+}\right\|_{L^{p}(\Omega)} \geq c & \forall u \in K_{1}, \\
\left\|\nabla u_{-}\right\|_{L^{p}(\Omega)} \geq c & \forall u \in K_{2}, \\
\left\|\nabla u_{+}\right\|_{L^{p}(\Omega)},\left\|\nabla u_{-}\right\|_{L^{p}(\Omega)} \geq c & \forall u \in K_{3} .
\end{aligned}
$$

Proof. By the definition of $K_{i}$,by (F3) and the Poincaré inequality we have that

$$
\begin{aligned}
\left\|\nabla u_{ \pm}\right\|_{L^{p}(\Omega)}^{p} & =\int_{\Omega} \lambda f(x, u) u_{ \pm}+\left|u_{ \pm}\right|^{p^{*}} d x \leq C\left\|u_{ \pm}\right\|_{L^{q}(\Omega)}^{q}+\left\|u_{ \pm}\right\|_{L^{p^{*}}(\Omega)}^{p^{*}} \\
& \leq c_{1}\left\|\nabla u_{ \pm}\right\|_{L^{p}(\Omega)}^{q}+c_{2}\left\|\nabla u_{ \pm}\right\|_{L^{p}(\Omega)}^{p^{*}} .
\end{aligned}
$$

As $p<q<p^{*}$, this finishes the proof.

Lemma 4. There exists $c>0$ such that $\Phi(u) \geq c\|\nabla u\|_{L^{p}(\Omega)}^{p}$ for every $u \in W^{1, p}(\Omega)$ if $\|u\|_{W_{0}^{1, p}(\Omega)}$ is small enough.

Proof. By (F3) and the Poincaré inequality we have

$$
\begin{aligned}
\Phi(u) & =\int_{\Omega} \frac{1}{p}|\nabla u|^{p}-\frac{1}{p^{*}}|u|^{p^{*}}-\lambda F(x, u) d x \\
& \geq \frac{1}{p}\|\nabla u\|_{L^{p}(\Omega)}^{p}-\frac{1}{p^{*}}\|u\|_{L^{p^{*}(\Omega)}}^{p^{*}}-C\|u\|_{L^{q}(\Omega)}^{q} \\
& \geq \frac{1}{p}\|\nabla u\|_{L^{p}(\Omega)}^{p}-C_{1}\left(\|\nabla u\|_{L^{p}(\Omega)}^{p^{*}}+\|\nabla u\|_{L^{p}(\Omega)}^{q}\right) \\
& \geq C\|\nabla u\|_{L^{p}(\Omega)},
\end{aligned}
$$

if $\|\nabla u\|_{L^{p}(\Omega)}$ is small enough, as $p<q<p^{*}$.

The following lemma describes the properties of the manifolds $M_{i}$. 
Lemma 5. $M_{i}$ is a $C^{1}$ sub-manifold of $W_{0}^{1, p}(\Omega)$ of co-dimension $1(i=1,2)$, 2 $(i=3)$ respectively. The sets $K_{i}$ are complete. Moreover, for every $u \in M_{i}$ we have the direct decomposition

$$
T_{u} W_{0}^{1, p}(\Omega)=T_{u} M_{i} \oplus \operatorname{span}\left\{u_{+}, u_{-}\right\},
$$

where $T_{u} M$ is the tangent space at $u$ of the Banach manifold $M$. Finally, the projection onto the first component in this decomposition is uniformly continuous on bounded sets of $M_{i}$.

Proof. Let us denote

$$
\begin{aligned}
& \bar{M}_{1}=\left\{u \in W_{0}^{1, p}(\Omega): \int_{\Omega} u_{+} d x>0\right\} \\
& \bar{M}_{2}=\left\{u \in W_{0}^{1, p}(\Omega): \int_{\Omega} u_{-} d x>0\right\}, \\
& \bar{M}_{3}=\bar{M}_{1} \cap \bar{M}_{2} .
\end{aligned}
$$

Observe that $M_{i} \subset \bar{M}_{i}$.

The set $\bar{M}_{i}$ is open in $W^{1, p}(\Omega)$, therefore it is enough to prove that $M_{i}$ is a $C^{1}$ submanifold of $\bar{M}_{i}$. In order to do this, we will construct a $C^{1}$ function $\varphi_{i}: \bar{M}_{i} \rightarrow \mathbb{R}^{d}$ with $d=1(i=1,2), d=2(i=3)$ respectively and $M_{i}$ will be the inverse image of a regular value of $\varphi_{i}$.

In fact, we define: For $u \in \bar{M}_{1}$,

$$
\varphi_{1}(u)=\int_{\Omega}\left|\nabla u_{+}\right|^{p}-\left|u_{+}\right|^{p^{*}}-\lambda f(x, u) u_{+} d x .
$$

For $u \in \bar{M}_{2}$,

$$
\varphi_{2}(u)=\int_{\Omega}\left|\nabla u_{-}\right|^{p}-\left|u_{-}\right|^{p^{*}}-\lambda f(x, u) u_{-} d x
$$

For $u \in \bar{M}_{3}$,

$$
\varphi_{3}(u)=\left(\varphi_{1}(u), \varphi_{2}(u)\right) .
$$

Obviously, we have $M_{i}=\varphi_{i}^{-1}(0)$. From standard arguments (see [9], or the appendix of [19]), $\varphi_{i}$ is of class $C^{1}$. Therefore, we only need to show that 0 is a regular value for $\varphi_{i}$. To this end we compute, for $u \in M_{1}$,

$$
\begin{aligned}
\left\langle\nabla \varphi_{1}(u), u_{+}\right\rangle & =p\left\|\nabla u_{+}\right\|^{p}-p^{*}\left\|u_{+}\right\|^{p^{*}}-\lambda \int_{\Omega} f(x, u) u_{+}-f_{u}(x, u) u_{+}^{2} d x \\
& \leq p^{*}\left(\left\|\nabla u_{+}\right\|^{p}-\left\|u_{+}\right\|^{p^{*}}\right)-\lambda \int_{\Omega} f(x, u) u_{+}-f_{u}(x, u) u_{+}^{2} d x \\
& \leq\left(p^{*} \lambda-\lambda\right) \int_{\Omega} f(x, u) u_{+} d x-\int_{\Omega} f_{u}(x, u) u_{+}^{2} d x .
\end{aligned}
$$

By (F3) the last term is bounded by

$$
\begin{aligned}
\left(p^{*} \lambda-\lambda-\frac{\lambda}{c_{1}}\right) \int_{\Omega} f(x, u) u_{+} d x & =\left(p^{*}-1-\frac{1}{c_{1}}\right)\left(\left\|\nabla u_{+}\right\|_{L^{p}(\Omega)}^{p}-\left\|u_{+}\right\|_{L^{p^{*}}(\Omega)}^{p^{*}}\right) \\
& \leq\left(p^{*}-1-\frac{1}{c_{1}}\right)\left\|\nabla u_{+}\right\|_{L^{p}(\Omega)}^{p} .
\end{aligned}
$$


Recall that $c_{1}<1 /\left(p^{*}-1\right)$. Now, the last term is strictly negative by Lemma 3 , Therefore, $M_{1}$ is a $C^{1}$ sub-manifold of $W^{1, p}(\Omega)$. The exact same argument applies to $M_{2}$. Since trivially

$$
\left\langle\nabla \varphi_{1}(u), u_{-}\right\rangle=\left\langle\nabla \varphi_{2}(u), u_{+}\right\rangle=0
$$

for $u \in M_{3}$, the same conclusion holds for $M_{3}$.

To see that $K_{i}$ is complete, let $u_{k}$ be a Cauchy sequence in $K_{i}$, then $u_{k} \rightarrow u$ in $W^{1, p}(\Omega)$. Moreover, $\left(u_{k}\right)_{ \pm} \rightarrow u_{ \pm}$in $W^{1, p}(\Omega)$. Now it is easy to see, by Lemma 3 and by continuity that $u \in K_{i}$.

Finally, by the first part of the proof we have the decomposition

$$
T_{u} W^{1, p}(\Omega)=T_{u} M_{i} \oplus \operatorname{span}\left\{u_{+}\right\}
$$

Where $M_{1}=\left\{u: \varphi_{1}(u)=0\right\}$ and $T_{u} M_{1}=\left\{v:\left\langle\nabla \varphi_{1}(u), v\right\rangle=0\right\}$. Now let $v \in T_{u} W_{0}^{1, p}(\Omega)$ be a unit tangential vector, then $v=v_{1}+v_{2}$ where $v_{2}=\alpha u_{+}$and $v_{1}=v-v_{2}$. Let us take $\alpha$ as

$$
\alpha=\frac{\left\langle\nabla \varphi_{1}(u), v\right\rangle}{\left\langle\nabla \varphi_{1}(u), u_{+}\right\rangle} .
$$

With this choice, we have that $v_{1} \in T_{u} M_{1}$. Now

$$
\left\langle\varphi_{1}(u), v_{1}\right\rangle=0 .
$$

The very same argument to show that $T_{u} W^{1, p}(\Omega)=T_{u} M_{2} \oplus\left\langle u_{-}\right\rangle$and $T_{u} W^{1, p}(\Omega)=$ $T_{u} M_{3} \oplus\left\langle u_{+}, u_{-}\right\rangle$.

From these formulas and from the estimates given in the first part of the proof, the uniform continuity of the projections onto $T_{u} M_{i}$ follows.

Now, we need to check the Palais-Smale condition for the functional $\Phi$ restricted to the manifold $M_{i}$. To this end, we need the following lemma from [16 which proves the Palais-Smale condition for the unrestricted functional below certain energy level.

Lemma 6 (J. García-Azorero, I. Peral, [16). Let $S_{p}$ be the best Sobolev constant

$$
S_{p}:=\inf _{\phi \in C_{c}^{\infty}\left(\mathbb{R}^{N}\right)} \frac{\int_{\mathbb{R}^{N}}|\nabla \phi|^{p} d x}{\left(\int_{\mathbb{R}^{N}}|\phi|^{p^{*}} d x\right)^{p / p^{*}}} .
$$

Then, the unrestricted functional $\Phi$ verifies the Palais-Smale condition for energy level c for every $c<\frac{1}{N} S_{p}^{N / p}$.

The proof of Lema 6 is based on the concentration compactness method.

Now, we can prove the Palais-Smale condition for the restricted functional.

Lemma 7. The functional $\left.\Phi\right|_{K_{i}}$ satisfies the Palais-Smale condition for energy level c for every $c<\frac{1}{N} S_{p}^{N / p}$.

Proof. Let $\left\{u_{k}\right\} \subset K_{i}$ be a Palais-Smale sequence, that is $\Phi\left(u_{k}\right)$ is uniformly bounded and $\left.\nabla \Phi\right|_{K_{i}}\left(u_{k}\right) \rightarrow 0$ strongly. We need to show that there exists a subsequence $u_{k_{j}}$ that converges strongly in $K_{i}$.

Let $v_{j} \in T_{u_{j}} W_{0}^{1, p}(\Omega)$ be a unit tangential vector such that

$$
\left\langle\nabla \Phi\left(u_{j}\right), v_{j}\right\rangle=\left\|\nabla \Phi\left(u_{j}\right)\right\|_{W^{-1, p^{\prime}}(\Omega)} .
$$

Now, by Lemma 5, $v_{j}=w_{j}+z_{j}$ with $w_{j} \in T_{u_{j}} M_{i}$ and $z_{j} \in \operatorname{span}\left\{\left(u_{j}\right)_{+},\left(u_{j}\right)_{-}\right\}$. 
Since $\Phi\left(u_{j}\right)$ is uniformly bounded, by Lemma $2, u_{j}$ is uniformly bounded in $W_{0}^{1, p}(\Omega)$ and hence $w_{j}$ is uniformly bounded in $W_{0}^{1, p}(\Omega)$. Therefore

$$
\left\|\nabla \Phi\left(u_{j}\right)\right\|_{W^{-1, p^{\prime}}(\Omega)}=\left\langle\nabla \Phi\left(u_{j}\right), v_{j}\right\rangle=\left\langle\left.\nabla \Phi\right|_{K_{i}}\left(u_{j}\right), v_{j}\right\rangle \rightarrow 0 .
$$

As $w_{j}$ is uniformly bounded and $\left.\nabla \Phi\right|_{K_{i}}\left(u_{k}\right) \rightarrow 0$ strongly, the inequality converges strongly to 0 . Now the result follows by Lema 6 .

We now immediately obtain

Lemma 8. Let $u \in K_{i}$ be a critical point of the restricted functional $\left.\Phi\right|_{K_{i}}$. Then $u$ is also a critical point of the unrestricted functional $\Phi$ and hence a weak solution to $(\mathrm{P})$.

With all this preparatives, the proof of the Theorem follows easily.

Proof of Theorem 1. To prove the Theorem, we need to check that the functional $\left.\Phi\right|_{K_{i}}$ verifies the hypotheses of the Ekeland's Variational Principle [6].

The fact that $\Phi$ is bounded below over $K_{i}$ is a direct consequence of the construction of the manifold $K_{i}$.

Then, by Ekeland's Variational Principle, there existe $v_{k} \in K_{i}$ such that

$$
\Phi\left(v_{k}\right) \rightarrow c_{i}:=\inf _{K_{i}} \Phi \quad \text { and } \quad\left(\left.\Phi\right|_{K_{i}}\right)^{\prime}\left(v_{k}\right) \rightarrow 0 .
$$

We have to check that if we choose $\lambda$ large, we have that $c_{i}<\frac{1}{N} S_{p}^{N / p}$. This follows easily from Lemma 1. For instance, for $c_{1}$, we have that choosing $w_{0} \geq 0$,

$$
c_{1} \leq \Phi\left(t_{\lambda} w_{0}\right) \leq \frac{1}{p} t_{\lambda}^{p} \int_{\Omega}\left|\nabla w_{0}\right|^{p} d x
$$

Hence $c_{1} \rightarrow 0$ as $\lambda \rightarrow 0$. Moreover, it follows from the estimate of $t_{\lambda}$ in Lemma 1 that $c_{i}<\frac{1}{N} S_{p}^{N / p}$ for $\lambda>\lambda^{*}\left(p, q, n, c_{3}\right)$. The other cases are similar.

From Lemma [6, it follows that $v_{k}$ has a convergent subsequence, that we still call $v_{k}$. Therefore $\Phi$ has a critical point in $K_{i}, i=1,2,3$ and, by construction, one of them is positive, other is negative and the last one changes sign.

\section{REFERENCES}

[1] D. Arcoya and J.I. Diaz. S-shaped bifurcation branch in a quasilinear multivalued model arising in climatology. J. Differential Equations, 150 (1998), 215-225.

[2] C. Atkinson and K. El Kalli. Some boundary value problems for the Bingham model. J. Non-Newtonian Fluid Mech. 41 (1992), 339-363.

[3] C. Atkinson and C.R. Champion. On some boundary value problems for the equation $\nabla(F(|\nabla w|) \nabla w)=0$. Proc. R. Soc. London A, 448 (1995), 269-279.

[4] T. Bartsch and Z. Liu. On a superlinear elliptic p-Laplacian equation. J. Differential Equations, 198 (2004), 149-175.

[5] S. Cingolani and G. Vannella, Multiple positive solutions for a critical quasilinear equation via Morse theory, Ann. Inst. H. Poincaré Anal. Non Linéaire, doi:10.1016/j.anihpc.2007.09.003.

[6] I. Ekeland. On the variational principle. J. Math. Anal.Appl., Vol 47 (1974), 324-353.

[7] M. del Pino and C. Flores. Asymptotic behavior of best constants and extremals for trace embeddings in expanding domains. Comm. Partial Differential Equations, 26 (11-12) (2001), 2189-2210.

[8] J.I. Diaz. Nonlinear partial differential equations and free boundaries. Pitman Publ. Program 1985. 
[9] G. Dinca, P. Jebelean and J. Mawhin Variational and Topological methods for Dirichlet Problems with p-Laplacian Portugalie Mathematica. Vol. 58, No. 3, pp. 339-378 (2001)

[10] J. F. Escobar, Uniqueness theorems on conformal deformations of metrics, Sobolev inequalities, and an eigenvalue estimate. Comm. Pure Appl. Math., 43 (1990), 857-883.

[11] J. Fernández Bonder. Multiple positive solutions for quasilinear elliptic problems with signchanging nonlinearities. Abstr. Appl. Anal., 2004 (2004), no. 12, 1047-1056

[12] J. Fernández Bonder and J.D. Rossi. Existence results for the p-Laplacian with nonlinear boundary conditions. J. Math. Anal. Appl., 263 (2001), 195-223.

[13] J. Fernández Bonder and J.D. Rossi. Asymptotic behavior of the best Sobolev trace constant in expanding and contracting domains. Comm. Pure Appl. Anal. 1 (2002), no. 3, 359-378.

[14] J. Fernández Bonder, E. Lami-Dozo and J.D. Rossi. Symmetry properties for the extremals of the Sobolev trace embedding. Ann. Inst. H. Poincaré Anal. Non Linèaire, 21 (2004), no. 6, 795-805.

[15] J. Fernández Bonder, S. Martínez and J.D. Rossi. The behavior of the best Sobolev trace constant and extremals in thin domains. J. Differential Equations, 198 (2004), no. 1, 129148.

[16] J. Garcia-Azorero and I. Peral, Multiplicity of solutions for elliptic problems with critical exponent or with a nonsymmetric term. Trans. Amer. Math. Soc. 323 (1991), no. 2, 877-895.

[17] M. Guedda and L. Veron, Quasilinear elliptic equations involving critical Sobolev exponents, Nonlinear Anal. T.M.A. 13 (1989), 879-902.

[18] P.L. Lions. The concentration-compactness principle in the calculus of variations. The limit case, part 1, Rev. Mat. Iberoamericana. Vol. 1 No.1 (1985), 145-201

[19] P. Rabinowitz, Minimax methods in critical point theory with applications to differential equations, CBMS Regional Conf. Ser. in Math., no. 65, Amer. Math. Soc., Providence, R.I. (1986).

[20] J.T. Schwartz. Generalizing the Lusternik-Schnirelman theory of critical points. Comm. Pure Appl. Math., 17 (1964), 307-315.

[21] M. Struwe. Three nontrivial solutions of anticoercive boundary value problems for the Pseudo-Laplace operator. J. Reine Angew. Math. 325 (1981), 68-74.

[22] P. Tolksdorf. Regularity for a more general class of quasilinear elliptic equations. J. Differential Equations, 51 (1984), 126-150.

[23] Z. Zhang, J. Chen and S. Li. Construction of pseudo-gradient vector field and sign-changing multiple solutions involving p-Laplacian. J. Differential Equations, 201 (2004), 287-303.

Pablo De Nápoli

Departamento de Matemática, FCEyN

UBA (1428) Buenos Aires, Argentina.

E-mail address: pdenapo@dm.uba.ar

Web-page: http://mate.dm.uba.ar/ pdenapo

JuLián Fernández Bonder

Departamento de Matemática, FCEyn

UBA (1428) Buenos Aires, Argentina.

E-mail address: jfbonder@dm.uba.ar

Web-page: http://mate.dm.uba.ar/ jfbonder

Analía Silva

Departamento de Matemática, FCEyN

UBA (1428) Buenos Aires, Argentina.

E-mail address: asilva@dm.uba.ar 